orange-red larvæ are gregarious internal feeders, which tunnel in companies through the tissues of the plant, thus providing also for the ingress of disease organisms. In this manner the pricklypear ultimately becomes so completely destroyed that it is reduced to a rotting mass of pulp.

The various other insects that were established have either become suppressed, or their activities nullified, owing to competition with the Cactoblastis. It is only locally, and in relation to a few Opuntia species of minor importance, that the Cactoblastis has shown itself to be ineffective. The most serious of these plants is Opuntia aurantiaca, but there is good reason to believe that its control by a species of Dactylopius (cochineal), also from the Argentine, seems assured.

The results of the repression programme have led to $22,000,000$ acres of former dense pear country in Queensland being selected for settlement. The previous value of this land was almost nothing, but freed from the pest its capital value would average $10 \mathrm{~s}$. an acre, without taking into consideration the worth of the new improvements in fencing, water facilities, removal of timber, etc. Hence the State has gained an asset of at least $£ 10,000,000$ because of the availability for farming of land hitherto useless. The area once under impenetrable prickly-pears, and now converted to dairy pastures, must exceed $1,000,000$ acres on which many hundreds of new settlers live. In New South Wales the greater part of the former prickly-pear country has been brought into production, mainly for sheep grazing, and most of it has been utilized to enlarge adjacent pastoral properties.

The present status of Cactoblastis is satisfactory. Concentrated numbers of larvæ are still attacking, reducing and destroying many of the remaining prickly-pear areas of heavier growth. Native parasitic Hymenoptera are not exercising an undue amount of control and their importance is not growing. After an interesting discussion of the future of the problem, Mr. Dodd concludes that, up to date, there is no indication that prickly-pear will not continue to be held in complete subjugaticn by its remarkably efficient lepidopterous enemy.

\title{
THUNDERSTORM PROBLEMS
}

\section{By DR. F. J. W. Whipple}

$\mathrm{O}^{\mathrm{N}}$ July 20 the London Branch of the Institute of Physics visited the Royal Meteorological Society at South Kensington to take part in a discussion on "Thunderstorm Problems". Sir George Simpson, president of the Royal Meteorological Society, was in the chair-a happy arrangement, for Sir George has been working at thunderstorms for nearly forty years; his classical work in India, which included the experiments on which the breaking-drop theory of the production of electricity is based, was published so long ago as 1909 and his most recent papers on the subject describe work of equal importance.

The discussion was opened by Dr. T. E. Allibone, who spoke on "The Physics of Lightning". His survey began with the year 1889, when Vernon Boys told the Physical Society that a lightning flash consisted of many separate strokes traversing the same path. Later, Hoffert recorded eight strokes on a photographic plate by waggling the camera about a vertical axis, and Walter, by using a camera mounted on a turn-table, was able to show that the strokes might be spread over a period of a second. It was Boys again who constructed a camera incorporating two identical lenses rotating about a common axis. The two images of a flash photographed with this camera were distorted differently and, the rate of rotation of the lenses being known, the speed with which the flash had developed could be determined. The Boys camera has been used effectively by Schonland in his studies of storms in the neighbourhood of Johannesburg.

Schonland and his collaborators demonstrated that a lightning flash to earth is a very complicated phenomenon. There is a preliminary 'leader' which starts from the cloud and sends out a number of branches; when one of these reaches the ground it becomes the channel for the main stroke up which the luminosity travels from ground to cloud. Subsequent leaders and main strokes use the same channel. The first leader alone is branched. Its progress is by jerky steps of about 50 metres. Comparison with electrical records has shown that the current in the main stroke is a hundred or more times as strong as that in the leader stroke. The main stroke removes to the ground the charges lowered from the cloud by the leader and retained for a while as ionization in the channel. It may be noted that in detailed studies the timing of a leader is conveniently reckoned in milliseconds, that of the progress of the main stroke in microseconds, while the intervals between the strokes are a few hundredths of a second. 
Schonland's work has been supplemented by that of McEachron, who photographed lightning striking the Empire State Building in New York and other lofty structures. The summit of the Empire State Building is 1,250 ft. above ground. McEachron discovered that, when such a high building is struck, there is an inversion of Schonland's initial process and a stepped leader travels upwards from the building. This leader is not followed at once by a main stroke; a dart leader from cloud to building intervenes. The subsequent strokes constituting the flash are of the normal type. At Pittsburgh, where oscillograms were taken showing the character of the lightning striking a tower $500 \mathrm{ft}$. high, it was found that the first leaders could be either upwards or downwards.

The most powerful currents in a lightning flash occur in the successive main strokes. The currents reach maxima of the order 20,000 amperes and each main stroke lowers a fraction of a coulomb of charge to the ground. This charge comes from the channel ionized by the leader, but many large quantities of electricity are brought down by continuing flow following the main strokes. The total discharge from a flash varies from two or three coulombs up to 100 or more. The most frequent value is about 20 coulombs.

Finally, Dr. Allibone sketched briefly theories which have been suggested in explanation of the jerky movement of leader strokes. He believes that laboratory experiments with camera and oscillograph may throw light on such theories. The potential gradient at which a flash can start has yet to be calculated.

The next subject to be considered, the distribution of electricity in thunderclouds, was introduced by Dr. G. D. Robinson. Until recently there were wide differences of opinion on this subject. The evidence available consisted of observations of potential gradient near the ground and of estimates of the charges brought down by lightning. The evidence was interpreted in various ways. To overcome the difficulties of the subject, it was necessary to develop some form of electrometer which could be carried by a balloon and sent up into the clouds. A suitable instrument, the altielectrograph, was developed at Kew Observatory. The records obtained with this instrument indicate variations of the sign of the potential gradient by the alternation of the traces of a pair of points on a rotating sheet of prepared paper. Changes in magnitude of the gradient affect the width of the traces and exceptionally strong gradients may cause sparking through the paper.

The discussion, by Sir George Simpson and F. J. Scrase, of the data obtained with the alti-electrographs sent up from Kew in 1935 and 1936 , led to the conclusion that in the typical summer thunder- storm of south-east England there is a concentration of positive electricity at the top of the cloud, a concentration of negative electricity lower down and another less widespread concentration of positive electricity near the base of the cloud. Further, it was noticed that the concentration of negative charge is always centred at a level at which the temperature is below the freezing-point. The evidence was clear that two processes were at work, one where the cloud held snow and hail, the other where the precipitation had taken the form of raindrops. The positive charge concentrated near the base of the cloud could be explained by the breaking of the larger raindrops in accordance with the theory propounded by Simpson himself in 1909. It was recognized that the process which was active in the upper part of the cloud was analogous to the electrification of drifting snow. Collisions of ice crystals charge the ice negatively and the air positively. It is the settling of the ice crystals through the air which separates the charges. Incidentally, it may be mentioned that C. T. R. Wilson pointed out in 1923 , in the "Dictionary of Applied Physics", that "Simpson's explanation of the electrical effects of drifting snow would seem to imply that snow and hail clouds should be of positive polarity", but this remark seems to have passed unnoticed.

Recently Sir George Simpson and Dr. Robinson have given details of additional soundings at Kew. They have discussed anew the earlier evidence and cleared up various difficulties. The triple structure of the summer thundercloud may be regarded as well established. The structure of thunderclouds in other parts of the world must be very similar, but it is likely that in some regions, notably in South Africa, where Schonland finds a great preponderance of negative charge brought down by lightning, the positive charge at the bottom of a cloud is not so well developed as in England.

In his contribution to the discussion, Mr. J. F. Shipley dealt with the development of thunderstorms and with the character of the air currents in the clouds. He has found evidence for the existence inside a thundercloud of great eddies, like smoke rings with a common vertical axis. That aeronauts who have ventured in balloons into thunderclouds have experienced violent movements, downwards as well as upwards, is notorious. Occasionally a balloon has been carried up and down repeatedly through several hundred feet. This is what would have happened if the balloon had entered a great vortex ring. The same hypothesis accounts for the appearance of some hailstones, which have grown like apples or like miniature fly-wheels. These must have been rotating violently during their growth. It must be admitted, however, that there is nothing in the appearance of 
most thunderclouds to suggest the presence of the vortex rings. Perhaps careful watching of accelerated motion pictures would reveal their existence.

Since the earliest days of wireless telegraphy it has been known that distant lightning affects the apparatus, and the hope has been entertained that it would be practicable to obtain direct observations which would be of use in locating storms with sufficient accuracy to meet the needs of the weather forecaster. Modern methods of recording and analysing atmospheries, the electromagnetic waves which proceed from lightning flashes, were explained to the meeting by Mr. F. E. Lutkin. The wave forms can be photographed with suitable apparatus, the horizontal movement of the light. spot of a cathode ray oscillograph being recorded on paper carried by a rapidly rotating drum. A high peripheral velocity, several metres per second, is needed.

There are a number of types of atmospheric. In the most frequent a series of oscillations with diminishing amplitude merges into a long smooth surge. The principal oscillations gradually lengthen, the first interval between peaks being of the order 150 microseconds and the fifth of the order 300 microseconds. The long surge lasts about a millisecond. The oscillatory movement is associated, it is believed, with the main stroke in a lightning flash, the long surge with the continuous discharge through the ionized channel.

In Australia and South Africa the peaks in the oscillatory movement have been related to the distance of origin by assuming each to be an echo, which has been subjected to a number of reflexions from the ionosphere and from the ground. In this way both the distance of the lightning and the effective height of the ionosphere can be determined.

The Australian and South African teams have both contended that the amplitude of the atmospherics can be used to measure the distance of origin. The approximation can only be very rough. The records at Slough show that the mean value of the field charge at the beginning of an atmospheric varies from 50 volts per metre at $25 \mathrm{~km}$. to 0.1 at $5,000 \mathrm{~km}$., but the individual values are well scattered about the means. A variation in the ratio of ten to one is often found in the amplitudes of atmospherics from a single source.

There are numerous applications of direction finding in the study of atmospherics. For locating storms two stations are required, preferably with telephonic communication; the various areas in which storms are taking place can be identified without difficulty and with reasonable precision.

The number of atmospherics received in Great Britain is greatest during the night in winter ; 100 per second is a rate of frequent occurrence. These originate chiefly in tropical America. In summer, similar sources are indicated during the night hours and, during the day, there are large impulses, occurring less frequently, of European origin. Conditions are never favourable for the trans. mission to England of atmospherics from Africa, for the thunderstorms occur there in our day-time, when long waves cannot be transmitted.

A lively general discussion followed the four formal contributions. The outstanding difficulty in the whole subject is to explain how a lightning stroke begins. The idea that, owing to the existence of a large space charge, potential gradient increases from the ground up to a maximum at the base of a thundercloud, a maximum high enough to initiate a spark, has had to be abandoned. Very strong gradients, sufficient to cause sparking in the alti-electrograph, occur however inside the clouds, and this suggests that a flash to ground originates within the cloud and extends downward in the leader stroke process. The suggestion was made in the discussion that the trigger might be a spark between two drops, highly charged with electricity of opposite signs, brought together in their fall through the cloud.

Much interest was shown in the location of thunderstorms by radio apparatus, and it was stated that a practical application of the method has been found in the safeguarding of transmission lines. It is sometimes possible to cut out of circuit a line which passes through a region where thunderstorms are active, the instructions being sent from a central station with apparatus for determining the bearings of atmospherics.

As to apparatus required for further studies of thunderstorm problems, perhaps the outstanding need is for the development of simple electrographs which will serve to provide measurements of the strength of the field in and around the clouds. More than one type of electrograph is needed, for weak fields have to be measured as well as strong ones. A comparison of records of strong fields will serve for a satisfactory calibration of the altielectrograph traces. The measurement of the weaker fields is especially desirable above the clouds. At present it is definitely known that the gradient immediately above a cloud is negative, so that an electric current starts upwards. Some of the lines of flow curve round and reach the ground at a modest distance; but it is believed that others go upwards to the ionosphere and carry electricity which is to return to earth in the airearth current of fine weather. It is to be hoped that, some day, measurements of potential gradient and conductivity will be obtained well above the clouds and show whether there is an upward current strong enough to furnish the appropriate quota of air-earth current. 